\title{
Programa de formación pedagógica a docentes orientado al aprendizaje cooperativo en estudiantes a través de Moodle
}

\author{
Andrea Lucía Córdoba Peralta ${ }^{1}$ \\ Herman Van de Velde ${ }^{2}$
}

\section{RESUMEN}

Artículo basado en los resultados de una investigación que la autora realizó con el objetivo de disponer de un programa de formación pedagógica a docentes, orientado al aprendizaje cooperativo en estudiantes a través del entorno virtual de aprendizaje (Moodle). El estudio constata que los entornos virtuales son medios educativos creados para el desarrollo de ambientes de aprendizaje, pero se necesita de una formación pedagógica para que esto suceda, por lo que los/as docentes requieren un programa que contemple esta temática. Se decidió trabajar con el enfoque cooperativo porque promueve la realización conjunta de actividades de aprendizaje entre estudiantes, respondiendo a las necesidades de una sociedad multicultural presente en las instituciones educativas. La contribución teórica del estudio consiste en la comprensión y promoción de la propuesta cooperativa como actividad interactiva entre estudiantes entre sí y con el/la docente desde un entorno virtual. Su aporte práctico implica el uso pedagógico de Moodle en los procesos educativos. Las novedades se relacionan con la utilización de las tecnologías y entornos virtuales como mediadores de los procesos de aprendizaje, la incorporación de técnicas didácticas y tecnológicas adaptadas a lo virtual, permitiendo a docentes contar con un espacio de formación pedagógica en dicho entorno.

Palabras clave: Entornos virtuales de aprendizaje, aprendizaje cooperativo, programa de formación pedagógica.

Recibido: 25 febrero de 2016

Aceptado: 10 de junio de 2016

1 Egresada de la Maestría en Pedagogía con mención en Docencia Universitaria. E-mail: aluciacp06@yahoo.com 2 Doctor en Ciencias Pedagógicas, docente UNAN-Managua / FAREM-Estelí y coordinador de ABACOenRed (www. abacoenred.com), en el contexto americano. Correo Electrónico: herman@abacoenred.com 


\title{
Teacher training program oriented to cooperative learning through Moodle
}

\begin{abstract}
This article is based on the results of a research work that aimed to arrange a teacher training program, oriented towards students' cooperative learning through the virtual learning platform Moodle. The study verifies that virtual platforms are educational means created for the development of learning environments; however, a pedagogical training is needed for those purposes. Hence, teachers need a program that meets this need. The cooperative approach was chosen since it promotes the joint performance of learning activities among students, satisfying the needs of a multicultural society present in educative institutions. The theoretical contribution of the study consists of the understanding and promotion of the cooperative proposal as the interactive activity among the students themselves, and with the teachers from a virtual environment. Its practical contribution implies the pedagogical use of Moodle in the educative processes. The contributions are related to the implementation of technologies and virtual platforms as mediators of the learning processes, the incorporation of didactic techniques and technologies adapted to the virtual, empowering teachers to count on a teacher training platform on MOODLE.
\end{abstract}

Keywords: Virtual learning environments, cooperative learning, teacher training. 


\section{INTRODUCCIÓN}

Las Tecnologías de la Información y Comunicación (TIC) han revolucionado al mundo entero y dan paso a la innovación educativa, que cada vez más requieren estar presentes en los procesos de formación profesional demandados por la sociedad actual. Como parte de estas tecnologías se encuentran los entornos virtuales de aprendizaje los cuales permiten la creación y desarrollo de cursos completos en la web. Para ello se necesita que docentes manejen de manera técnica y pedagógica dichos entornos.

Un entorno virtual de aprendizaje se presenta como un ámbito para promover el aprendizaje a partir de procesos de comunicación multidireccionales (docente/estudiante, estudiante/docente y estudiantes entre sí). Se trata de un ambiente de trabajo compartido para la construcción del conocimiento con base en la participación activa y la cooperación de todos los miembros del grupo. (Salinas, 2011, p. 2)

El/la docente de la modalidad virtual es quien dirige el proceso de aprendizaje de los y las estudiantes, según Lozano Castro (2010, p. 422):

El profesor que tiene experiencia en el ambiente de aprendizaje presencial o en el salón de clase tradicional deberá capacitarse en varios aspectos o temas claves para un mejor desempeño tales, como:

- ¿Qué son los cursos en línea?

- ¿Cómo aprende un estudiante de un curso en línea?

- Uso de la tecnología para la educación mediada por computadora

- El modelo educativo de la universidad o institución donde será tutor y las estrategias didácticas, componentes $\mathrm{y}$ recursos que conforman dicho modelo

- ¿Cómo retroalimentar al estudiante con calidad y calidez?
- Capacitación en los procesos docente, tecnológico, y administrativo con el fin de poder ser tutor de un curso.

Los entornos virtuales de aprendizaje ofrecen variadas posibilidades para la innovación. Salinas (2011, pp. 7-9) destaca las siguientes: "favorecer la adopción de un modelo centrado en el estudiante, extender los límites espacio-temporales del aula presencial, ampliar las oportunidades de comunicación, proponer nuevas estrategias metodológicas, propias del ámbito virtual y utilizar nuevos recursos didácticos".

Estas posibilidades mencionadas por Salinas coinciden con el Modelo Educativo de la Universidad Nacional Autónoma de Nicaragua, Managua (UNAN-Managua). El/la docente en su práctica educativa es el que va a hacer efectiva la innovación, al integrar el potencial que proporcionan las tecnologías en sus clases como apoyo al proceso de aprendizaje de estudiantes.

En cuanto al aprendizaje cooperativo y para efectos de este estudio, se construyó el siguiente concepto:

Es una estrategia de aprendizaje basada en la construcción colectiva de nuevas oportunidades de aprendizaje, caracterizadas por el ejercicio de:

- Responsabilidad compartida (compromiso)

- Poder compartido (co-gestión)

- Comunicación fluida y de calidad

- Transparencia (rendición de cuentas del trabajo realizado)

- Interdependencia positiva (confianza)

- Integración

- Crítica y auto-crítica constructiva.

Van de Velde (2014,p. 40) plantea que en la cooperación "hacemos énfasis en la intención de desarrollar un trabajo conjunto entre partes, tal que todas las involucradas se fortalezcan". Además, enfatiza en que la cooperación implica una manifestación actitudinal, caracterizada por la solidaridad en la construcción de soluciones de problemas compartidos. 
El aprendizaje cooperativo es un enfoque educativo ideal en la sociedad actual, procura el desarrollo personal y profesional de los/as estudiantes, quienes aprenden a valorar el trabajo que realizan con los/as integrantes del equipo, comparten experiencias de vida que elevan el aprendizaje propio y el/la de los/as demás. Es necesario recalcar que:

El aprendizaje cooperativo es la vía para alcanzar la calidad de la educación, que se logra cuando maestros/as y administrativos/as están dispuestos a trabajar y aprender juntos para redefinir políticas, reglamentos, normas y comportamientos escolares. Es la mejor respuesta a las condiciones históricas, socioeconómicas y culturales de la sociedad contemporánea. (Ferreiro Gravié \& Espino Calderón, 2009, p. 192)

Al referirse al 'aprendizaje cooperativo', cabe aclarar que no solo se refiere, en el contexto de este trabajo, a la aplicación de técnicas, sino a la construcción conjunta de escenarios educativos, caracterizados esencialmente por un ambiente global cooperativo.

El Modelo Educativo (2011, p. 32) de la UNANManagua plantea que:

En nuestra universidad se concibe el aprendizaje como un proceso interactivo, una actividad compartida en la que los/as estudiantes interactúan entre ellos/as, con el/la profesor/a y con todos los elementos que los rodean en el contexto sociocultural en que se desarrollan. De esta forma el trabajo cooperativo constituye uno de los pilares fundamentales del quehacer educativo.

La Facultad Regional Multidisciplinaria de Estelí (FAREM-Estelí) tiene incorporada en su página web una sección llamada 'Campus Virtual' para el servicio docente y como entono virtual de aprendizaje utiliza Moodle.
Los y las docentes trabajan con programas para el desarrollo de las asignaturas, ya que brindan la orientación de los contenidos que serán abordados, los objetivos que se quieren alcanzar en el proceso de formación de sus estudiantes y comparten algunas orientaciones metodológicas.

Los programas: "son conjuntos de acciones educativas cuya finalidad es atender las demandas y responder a las expectativas de las personas". (Asamblea General de la República de Nicaragua, 2006).

El empleo de la tecnología y la pedagogía facilita el proceso educativo del / de la docente y estudiantes al contar con herramientas interactivas que permiten el desarrollo de diferentes actividades como en el caso de los entornos virtuales.

Para usar un entorno virtual de aprendizaje y efectivamente innovar en las prácticas formativas en línea es necesaria la disponibilidad de los recursos tecnológicos, planes de formación del profesorado que consideren paradigmas metodológicos acordes a los nuevos tiempos, como el constructivismo y la construcción social del conocimiento y el desarrollo de competencias en el uso tecnológico de estos espacios y en las habilidades relacionadas con el rol docente como diseñador de experiencias formativas virtuales y animador de la interacción en estos espacios (Silva Quiroz, 2011, p. 66).

Las interacciones que se producen entre docente y estudiantes son resultado de la planificación de las actividades por parte del / de la docente. Deben procurarse intercambios de significados, aprendizajes a nivel personal y grupal, así como la construcción social del saber. Esto es válido para implementarse en ambientes presenciales y/o virtuales.

El uso de las TIC no son garantes de una práctica educativa de calidad, sino depende del grado de conocimientos y habilidades pedagógico- 
metodológicas del / de la docente al utilizar estas herramientas, como apoyo a la educación presencial o por medio de entornos virtuales de aprendizaje totalmente en línea, creando e impulsando nuevas propuestas y entornos educativos para el aprendizaje y la socialización de los/as estudiantes. Para ello se dispone de un programa de formación pedagógica.

\section{MATERIALES Y MÉTODOS}

Las herramientas metodológicas utilizadas en la planeación de la investigación realizada fueron la visión vertical (problema de investigación, preguntas directrices, objetivos, las personas involucradas en el estudio realizado y tipo de investigación) y la visión horizontal (contiene las principales interrogantes científicas de la investigación con las tareas, métodos, resultados y posible salida en la estructura final de la tesis)

La visualización del proceso de investigación se concreta en cuatro fases: planificación, recolección de datos, procesamiento de datos y la elaboración del informe final.

Con respecto a los métodos a nivel teórico se aplicó análisis, síntesis e inducción. Los primeros dos métodos permitieron realizar análisis documental, análisis y síntesis de contenido. También se implementaron en función de la interpretación de los resultados de la aplicación de los instrumentos de recolección de datos, para una mejor comprensión del uso que le dan docentes y estudiantes a los entornos virtuales de aprendizaje en el proceso educativo.

También se aplicó el método inductivo para extraer las conclusiones de carácter general de los hechos más repetitivos encontrados en los resultados obtenidos de la aplicación de instrumentos de recolección de datos a docentes.

Los métodos a nivel empírico empleados: encuesta, entrevista y observación. La primera técnica utilizada para la recolección de datos fue la encuesta por cuestionario con el objetivo de saber cómo los y las docentes trabajaban Moodle como entorno virtual de aprendizaje en sus clases. Posteriormente, para profundizar en los datos obtenidos del cuestionario, se aplicó la entrevista estructurada a docentes, con el objetivo de saber más respecto a cómo trabajan 'Moodle' como entorno virtual de aprendizaje, en sus clases. Finalmente se hizo la observación no participante al Campus Virtual de la FAREM-Estelí, construida según las respuestas obtenidas de las entrevistas a docentes, con el objetivo de valorar el diseño y organización de los cursos y sus ambientes educativos creados por docentes de la carrera de Ingeniería en Sistemas de Información al trabajar sus clases.

En esta investigación se emplearon dos métodos a nivel estadístico como fueron "promedio" para calcular la edad promedio de los/as docentes encuestados y "frecuencia" para representar los datos numéricos obtenidos del cuestionario, esto se debe a que el estudio es más cualitativo.

El enfoque paradigmático de esta investigación es mixto, predominando el enfoque cualitativo. En este estudio se empleó la triangulación de datos obtenidos de las técnicas aplicadas en el trabajo de campo (cuestionario y guía de entrevista a docentes, así como la guía de observación realizada al Campus Virtual de la FAREM-Estelí) y algunas teorías expuestas en el capítulo Referente teórico contextual.

En la selección de la muestra se escogió el muestreo no probabilístico, de tipo intencional o por conveniencia. Los criterios de selección de la muestra de la carrera "Ingeniería en Sistemas de Información" obedecen a:

- Docentes, en su mayoría, usan frecuentemente el laboratorio de computación

- La mayor parte de los y las docentes está sin preparación pedagógico-metodológica en el uso de entornos virtuales de aprendizaje

- Los/as estudiantes necesitan experienciar otras 
formas de aprendizajes, esto lo permiten los entornos virtuales

- Docentes con mayor posibilidad (por el tipo de carrera) de compartir datos útiles como insumos para la construcción de un programa de formación pedagógica a docentes.

La muestra elegida del estudio son 12 docentes (muestra invitada) de la carrera, de los cuales solamente 9 respondieron al llenado del cuestionario (muestra productiva).

\section{RESULTADOS Y DISCUSIÓN}

Se detallan los principales resultados obtenidos de la aplicación de técnicas de recolección de datos (cuestionario y entrevista estructurada a docentes y la observación no participante al Campus Virtual de la FAREM-Estelí), así como la triangulación de estos datos, y algunas teorías expresadas en el capítulo Referente teórico contextual.

De acuerdo a los resultados del cuestionario aplicado, los/as nueve docentes de la carrera de Ingeniería de Sistemas de Información realizan actividades académicas de forma presencial con sus estudiantes. Solamente cinco docentes mencionaron en sus respuestas que complementan las actividades presenciales con las virtuales a través del uso de Moodle. No hay algún seguimiento de docente a estudiantes, ni espacios creados en los cursos para aclaración de dudas. Se limitan a cumplir con entregas de tareas, siendo estas más de carácter evaluativo.

Las actividades planificadas por los/as docentes han de ser adecuadas "al contenido y a la caracterización del grupo, responder a las inquietudes que se les presenten, promover el aprendizaje cooperativo, la evaluación basada en criterios, motivarlos a que utilicen Moodle, fomentar la responsabilidad en el cumplimiento de entrega de tareas, la participación en las actividades orientadas" (expresiones tomadas de la entrevista a docentes), de manera que permitan la construcción de un entorno virtual de aprendizaje. Esto también es afirmado por lo que expresa Salgado García (2006, p. 69) desde el modelo constructivista las prácticas educativas en contraposición al modelo tradicional se caracterizan por fomentar "una relación más horizontal entre profesor/a y estudiante, la interacción entre estudiantes, el pensamiento crítico, la discusión, el intercambio de opiniones y construcciones sobre los fenómenos, la participación activa, las experiencias de aprendizaje y las evaluaciones auténticas basadas en tareas reales".

Se recalca que Moodle cuenta con un sinnúmero de opciones para la generación de aprendizajes y es necesario que sea atractivo a los/as participantes del mismo, pero requiere de los/as docentes propuestas de recursos didácticos que faciliten a los/as estudiantes la construcción de sus propios aprendizajes y puedan compartir con los/as demás integrantes del grupo.

En general se evidencia en el programa de formación solicitado por los/as docentes el disponer de toda una estructura organizativa en cuanto a diseño, recursos (internos y externos), actividades, técnicas de evaluación, en fin la creación de un entorno virtual de aprendizaje de forma pedagógica y didáctica para los/as estudiantes. Las estrategias del programa de formación están enfocadas a como los/as docentes trabajen en el diseño y aplicación de estrategias de aprendizaje en Moodle, desde una perspectiva interactiva, promoviendo espacios de reflexión en los cursos, la presentación de los contenidos a través de las pantallas, el intercambio entre todos/as los/as actores/as involucrados/as con herramientas propias de Moodle. También incluye la implementación del aprendizaje cooperativo en el proceso.

En cuanto a la metodología del programa de formación propuesta por los/as encuestados/as expresaron que esta debe ser "dinámica, interactiva, práctica, activa, debe permitir el compartir aprendizajes, flexible, tratar contenidos abiertos al debate y a la discusión”. 
El utilizar un entorno virtual que favorezca el aprendizaje cooperativo o no, estará definido por el diseño de las actividades pedagógicas en las cuales los/as estudiantes interactúan para construir sus aprendizajes y aportar al de los/as demás. Las metodologías cambian constantemente y es deber docente actualizarse de forma permanente, por lo que en sus respuestas manifiestan el interés en esta temática. Los y las docentes necesitan estar preparados/as ante los constantes cambios influenciados por las TIC, particularmente en la utilización de entornos virtuales de aprendizaje de forma técnica y pedagógica, orientando el aprendizaje cooperativo como elemento de innovación docente entre participantes y facilitador/a.

Para el desarrollo del programa de formación pedagógica a docentes se propuso un diplomado el cual está estructurado en tres módulos en el que se diferencian los niveles de participación docente (inicial, medio y avanzado), acordes a las características en las prácticas pedagógicas y partiendo de su situación inicial al ingresar al programa de formación. Estos módulos se distribuyen de la siguiente manera:

Módulo I: Uso y familiarización con el entorno virtual. Módulo II: Enfoque pedagógico-metodológico basado en aprendizaje cooperativo en el uso de un entorno virtual.

Módulo III: Creación de cursos virtuales usando un entorno virtual (Moodle), con enfoque pedagógico caracterizado por un aprendizaje cooperativo.

Los y las docentes participantes del diplomado ingresarán a los tres módulos del programa, el cual es visto como un solo curso. Habrá algunos/as que manejen más que otros/as dadas las experiencias diferenciadoras en cuanto al uso de Moodle.

\section{CONCLUSIONES Y RECOMENDACIONES}

El diagnóstico realizado sobre las prácticas pedagógicas refleja que quienes usaron el entorno
'Moodle' lo han hecho en mayor medida de manera técnica. Por lo mismo, este entorno no es aprovechado desde el punto de vista pedagógico, minimizando la importancia de este aspecto necesario para facilitar la construcción de aprendizajes con todos/as los/as actores/as involucrados/as en el proceso. Se destaca la débil incidencia docente en la promoción del trabajo cooperativo con sus estudiantes en los cursos desarrollados, desde el entorno virtual. La universidad cuenta con una sección Campus Virtual, sin embargo la participación de docentes en la creación de cursos como parte del proceso educativo para sus estudiantes, es limitada.

Los resultados de la revisión documental y su correspondiente análisis de contenido permiten fundamentar que los entornos virtuales de aprendizaje "son recursos educativos diseñados para facilitar la comunicación pedagógica de los/as participantes en un proceso educativo, sea a distancia (virtual) o de naturaleza mixta (combinación de lo presencial y virtual) mediatizados por la tecnología".

Se concluye que la metodología empleada, es decir el conjunto de métodos, técnicas e instrumentos que se aplicó permitió conseguir los alcances pretendidos y expresados en los objetivos de esta investigación.

En cuanto a los contenidos de un programa de formación pedagógica sugieren los/as docentes que sean novedosos, interactivos, prácticos y que les facilite el diseño de cursos virtuales. Retomando todos los insumos de los resultados se llegó a la construcción del programa de formación pedagógica, el cual será oportuno en función de las necesidades docentes en cuanto al manejo técnico y/o pedagógico de un entorno virtual de aprendizaje (Moodle).

Se recomienda desarrollar el programa de formación pedagógica en la carrera de Ingeniería en Sistemas de Información y validarlo. También se sugiere realizar diagnóstico en otras carreras para adecuar el programa 
de formación pedagógica y aplicarlo. La universidad podría establecer convenios que permitan a los/as docentes realizar un intercambio de experiencias con otras instituciones educativas que trabajan con entornos virtuales de aprendizaje. Además de lo anterior se sugiere documentar más técnicas cooperativas que se puedan desarrollar en entornos virtuales para el fortalecimiento de los procesos educativos. También se debe pensar en establecer un sistema de formación pedagógica a docentes en general de toda la Facultad y demás recintos de la UNAN-Managua.

\section{BIBLIOGRAFÍA}

Asamblea General de la República de Nicaragua. (2006). Ley General de Educación $N^{\circ} 582$. Managua.

Ferreiro Gravié, R., \& Espino Calderón, M. (2009). El ABC del Aprendizaje Cooperativo. Trabajo en equipo para aprender y enseñar (2da ed.). México: Trillas.

Lozano Castro, A. (2010). Prácticas de tutoría en educación a distancia. En A. Lozano Rodríguez, \& J. V. Burgos Aguilar, Tecnología Educativa en un modelo de educación a distancia centrado en la persona. México: Limusa.

Salgado García, E. (2006). Manual de Docencia Universitaria Introducción al constructivismo en la educación superior. (ULACIT, Ed.) Disponible en: http://cursa.ihmc.us/rid=1158251261031_49 9801323_3752/MANUAL\%20DOCENCIA\%20 UNIVERSITARIA\%202a\%20ed.pdf

Salinas, M. I. (2011). Entornos virtuales de aprendizaje en la escuela: tipos, modelo didáctico y rol del docente. Disponible en: http://www.uca.edu.ar/ uca/common/grupo82/files/educacion-EVA-en-laescuela_web-Depto.pdf

Silva Quiroz, J. (2011). Diseño y moderación de entornos virtuales de aprendizaje (EVA). Santiago de Chile: UOC.

Universidad Nacional Autónoma de Nicaragua, UNAN-Managua. (2 de Septiembre de 2011). Modelo Educativo, Normativa y Metodología para la Planificación Curricular 2011. Managua, Nicaragua.

Van de Velde, H. (2014). Construyendo escenarios educativos, basados en cooperación genuina (1 ra ed.). Estelí, Nicaragua: ABACOenRed. 